\title{
F-Actin Is Concentrated in Nonrelease Domains at Frog Neuromuscular Junctions
}

\author{
Anna Dunaevsky and Elizabeth A. Connor \\ Department of Biology, Neuroscience and Behavior Graduate Program, University of Massachusetts, Amherst, \\ Massachusetts 01003
}

To gain insight into the role of F-actin in the organization of synaptic vesicles at release sites, we examined the synaptic distribution of F-actin by using a unique synaptic preparation of frog target-deprived nerve terminals. In this preparation, imaging of the synaptic site was unobstructed by the muscle fiber cytoskeleton, allowing for the examination of hundreds of synaptic sites in their entirety in whole mounts. At target-deprived synaptic sites F-actin was distributed in a ladder-like pattern and was colocalized with $\beta$-fodrin. Surprisingly, F-actin stain, which we localized to the nerve terminal itself, did not overlap a synaptic vesicle marker, suggesting that it was concentrated in nonrelease domains of nerve terminals between clusters of synaptic vesicles. These findings suggest that the majority of the presynaptic $\mathrm{F}$-actin is not involved in tethering synaptic vesicles. Instead, the strategic presynaptic positioning of this cytoskeletal meshwork in nonrelease domains of the nerve terminal suggests alternate functions such as restricting synaptic vesicles to release domains, recycling synaptic vesicles, or stabilizing the nerve terminal.

Key words: F-actin; synaptic vesicles; cytoskeleton; presynaptic; $\beta$-fodrin; terminal Schwann cell
Synaptic vesicles are clustered at release sites in motor nerve terminals along with several molecules involved in their exocytosis (Scheller, 1995; Sudhof, 1995). This focusing of synaptic vesicles insures rapid and sustained release of neurotransmitter (Brodin et al., 1997). The synaptic vesicles at release sites have been described as two physically distinct populations (Kelly, 1993). One group of vesicles, docked at the presynaptic membrane, appear poised for release via interactions between vesicular and presynaptic membrane proteins (Sollner et al., 1993a,b). Another larger group of synaptic vesicles does not contact the presynaptic membrane and is clustered in the vicinity of release sites. These vesicles are thought to represent a reserve pool that is available for release during trains of stimuli (Betz and Bewick, 1992; Kelly, 1993). Photobleaching has demonstrated that the lateral mobility of synaptic vesicles within a cluster is restricted (Henkel et al., 1996; Kraszewski et al., 1996), yet the mechanism by which these vesicles are confined to release sites remains unclear. Any proposed mechanism must account for the localization of synaptic vesicles and also allow for vesicle movement during release and vesicle recycling.

A current model proposes that synaptic vesicles are tethered at release zones via phosphorylation-dependent interactions among the vesicular molecule, synapsin, and an actin-based cytoskeleton (Greengard et al., 1993). Although the role for synapsin in focusing synaptic vesicles at release sites appears well supported by both in vitro and in vivo experimental evidence (Greengard et al., 1993; Li et al., 1995; Pieribone et al., 1995; Rosahl et al., 1995; Takei et al., $1995)$, the contribution of actin filaments (F-actin) to this process is less clear. Although F-actin is concentrated in newly formed synaptic contacts in vitro (Dai and Peng, 1996; Wang et al., 1996;

\footnotetext{
Received Feb. 4, 2000; revised May 22, 2000; accepted May 25, 2000.
}

This work was supported by a University Graduate Fellowship to A.D. and a University of Massachusetts Faculty Research Grant and National Science Foundation Grant IBN-9602136 to E.A.C. We thank Shaunak Desai, Thomas Jaquith, Susan Kralian, Frank Marrero, and Hieu Nguyen for their contributions to these experiments. We also thank Pat Wadsworth and Rod Murphey for helpful advice in preparing this manuscript. Mount Holyoke College generously allowed us to use its $\mathrm{x}$-irradiation facilities.

Correspondence should be addressed to Dr. Elizabeth A. Connor, Department of Biology, University of Massachusetts, Amherst, MA 01003. E-mail: econnor@bio.umass.edu.

Dr. Dunaevsky's present address: Department of Pathology, Columbia University, Room 14-509, 630 West 168th Street, New York, NY 10032

Copyright (C) 2000 Society for Neuroscience $0270-6474 / 00 / 206007-06 \$ 15.00 / 0$
Bernstein et al., 1998), the demonstration of F-actin at mature synaptic release sites resulted in conflicting evidence (Landis et al., 1988; Hirokawa et al., 1989). Further, the release and recycling of synaptic vesicles as well as their distribution at mature vertebrate synapses were unaltered by treatment with agents that prevent F-actin polymerization (Betz and Henkel, 1994; Henkel et al., 1996; Job and Lagnado, 1998). Only okadaic acid, a phosphatase inhibitor, has been found to disrupt vesicle clusters (Betz and Henkel, 1994; Dai and Peng, 1996).

In the study presented here we used a unique synaptic preparation of frog target-deprived nerve terminals to examine the synaptic distribution of F-actin. In this preparation the resulting targetdeprived nerve terminals allow for a light-level whole-mount analysis of the presynaptic cytoskeleton at a mature, functional synapse. We previously demonstrated that frog motor nerve terminals are maintained functionally and structurally, often in their entirety, in the absence of muscle fibers (Dunaevsky and Connor, 1995, 1998). We report here that F-actin is distributed in a ladderlike pattern at target-deprived synaptic sites. Our analysis supports the localization of F-actin to the nerve terminal itself. Interestingly, the majority of the F-actin is excluded from release sites of the nerve terminal and thus is not involved in tethering synaptic vesicles. The strategic presynaptic positioning of this cytoskeletal meshwork in nonrelease domains of the nerve terminal is significant because the actin-based network may participate in restricting synaptic vesicles to release domains, in recycling synaptic vesicles, or in stabilizing the nerve terminal at a synaptic site.

\section{MATERIALS AND METHODS}

Surgery. Adult male frogs, Rana pipiens (Hazen, Alburg, VT), were anesthetized by immersion in $0.2 \%$ tricaine methane sulfonate, $\mathrm{pH} 7.2$, and cooled on ice. Cutaneous pectoris muscle fibers were damaged without injury to the motor innervation, as previously described (Dunaevsky and Connor, 1995). Within 2 weeks the muscle fibers degenerated and were phagocytized, leaving empty basal lamina sheaths of muscle fibers with original nerve terminals intact. These preparations are referred to as innervated sheaths. Muscle fiber regeneration was prevented by $\mathrm{x}$-irradiation of the thorax on $3 \mathrm{~d}$ consecutively at the time of surgery (Sanes et al., 1978; Yao, 1988). Innervated sheaths occasionally contained muscle fibers that either persisted after incomplete surgical damage or had regenerated. Synaptic sites in innervated sheaths were determined to be target-deprived by the absence of striations in bright-field microscopy or by a phalloidin-stained muscle fiber in fluorescence microscopy (Dunaevsky and Connor, 1995). In some instances the innervation was severed at the 


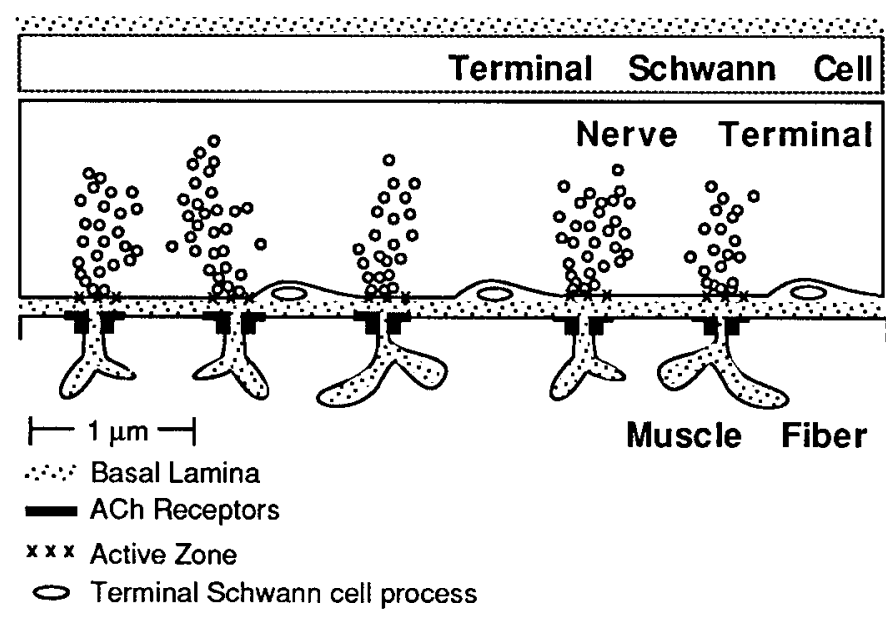

Figure 1. Longitudinal view through a region of a frog neuromuscular junction. The nerve terminal, capped by a terminal Schwann cell, is separated from the muscle membrane by the synaptic basal lamina. Several release sites in the nerve terminal are filled with synaptic vesicles and are aligned with junctional folds in the muscle membrane. Processes of the terminal Schwann cell encircle the nerve terminal in some nonrelease domains.

level of the brachial nerve exiting the vertebral column (Letinsky et al., 1976).

Staining of synaptic sites. Muscles or innervated sheaths (1-3 months after muscle damage) were dissected in normal frog Ringer's solution, fixed in either $1 \%$ formalin or $2 \%$ paraformaldehyde, and rinsed in $0.1 \%$ Triton X-100 in PBS (PBST; Connor et al., 1994). After a 10 min incubation in blocking solution (Connor et al., 1994) the preparations were incubated in primary antibody or fluorochrome-tagged probes for $1 \mathrm{hr}$ at room temperature or overnight at $4{ }^{\circ} \mathrm{C}$. Next the preparations were rinsed in PBST, incubated for 1-2 $\mathrm{hr}$ in fluorochrome-labeled secondary antibodies, and washed in PBST. Preparations were mounted on slides with an antifade reagent, Slow Fade-Light (Molecular Probes, Eugene, OR).

Antibodies and probes. F-actin was marked with fluorochromeconjugated phalloidin (Molecular Probes). Synaptic sites were identified either by staining acetylcholine receptors with $\alpha$-bungarotoxin or by staining synaptic and terminal Schwann cell basal laminae with peanut agglutinin (Ko, 1987; Dunaevsky and Connor, 1995). Primary antibodies included rabbit polyclonal antibodies against brain $\beta$-fodrin and brain $\alpha$-fodrin (gift of R. Bloch, University of Maryland School of Medicine) (Porter et al., 1997; Z hou et al., 1998); a mouse IgG monoclonal antibody $(\mathrm{mAb})$ directed against a synaptic vesicle antigen, SV2 (gift of S. Carlson, University of Washington); and mouse IgM mAbs (SC-1 and 2A12, gift of C-P Ko, University of Southern California) directed against epitopes that mark the surface of terminal Schwann cells and their basal laminae (Tyner et al., 1996; Astrow et al., 1999).

Imaging. Confocal images were collected with a Bio-Rad MRC 600 (Hercules, CA) mounted on a Nikon Optiphot with a $60 \times$ oil immersion objective. For each synaptic site $6-45$ sections were collected every $0.2 \mu \mathrm{m}$, with each being a Kalman average of four to five scans. Double-labeled preparations were viewed by using the dual wavelength mode (488 and 568 $\mathrm{nm}$ ). Images were pseudocolored, using National Institutes of Health Image software; adjustments to brightness and contrast as well as the merging of images were performed with Adobe Photoshop.

To compare the distribution of two stains at a given synaptic site in the $z$-plane, we resampled the volume data from a $z$-series of confocal images collected in the dual wavelength mode along a specified line. The integration of line scans through the $z$-axis yielded a longitudinal view of the synaptic site. These reconstructed line scans were pseudocolored and then merged as described above.

Distance measurements. Measurements were made by using National Institutes of Health Image software to determine the distance between adjacent phalloidin-stained bands. The center-to-center distance between adjacent SV2-stained clusters of synaptic vesicles was determined also.

\section{RESULTS}

\section{Organization of the frog neuromuscular junction}

The presynaptic component of the frog neuromuscular junction consists of an arborized nerve terminal capped by nonmyelinating terminal Schwann cells. The linear array of release sites at this synapse is marked by clusters of synaptic vesicles that appose junctional folds in the muscle membrane (Fig. 1). The release sites are separated by nonrelease domains that are generally free of vesicles. In these areas a cytoplasmic finger of a terminal Schwann

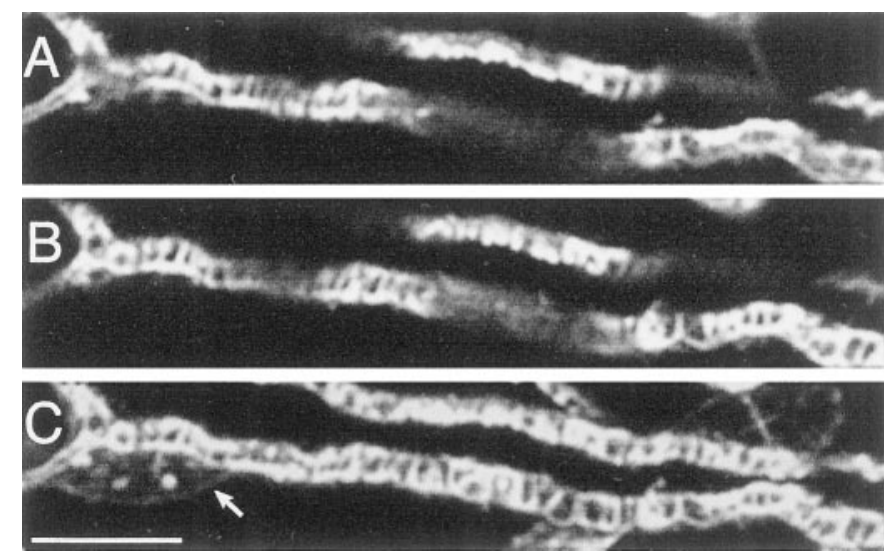

Figure 2. A target-deprived synaptic site is stained by phalloidin in a ladder-like pattern. $A, B$, Two confocal $z$-sections of a target-deprived nerve terminal stained with rhodamine-conjugated phalloidin to mark F-actin. $C$ A projection of a $z$-section series of 42 images. A terminal Schwann cell nucleus is marked with an arrow. Scale bar, $10 \mu \mathrm{m}$.

cell occasionally encircles the nerve terminal, intervening between the nerve terminal and the synaptic basal lamina (McMahan et al., 1972). These morphological features of the nerve terminal as well as the physical relationship between the nerve terminal and the terminal Schwann cell are maintained for months after damage and complete degeneration of the target muscle fiber (Yao, 1988; Dunaevsky and Connor, 1998). Target-deprived nerve terminals also release and recycle synaptic vesicles in response to stimulation (Dunaevsky and Connor, 1995).

\section{F-actin bands do not colocalize with clusters of synaptic vesicles}

To determine the presynaptic distribution of F-actin at a mature synapse, we stained innervated sheaths with fluorochromeconjugated phalloidin and viewed them in whole mount. In these preparations the connective tissue cells and muscle fibers were stained by phalloidin (data not shown) as were the synaptic sites. Although it was not possible to distinguish synaptic sites on persisting phalloidin-stained muscle fibers, target-deprived synaptic sites were visualized clearly. There, the F-actin stain was distributed in a ladder-like pattern; the longitudinal borders of the synaptic site were stained as well as bands that crossed the synaptic site like rungs of a ladder (Fig. 2).

To determine the relationship between the bands of F-actin stain and release sites, we stained innervated sheaths with phalloidin and an SV2 antibody that marked synaptic vesicles. The bands of actin stain in these preparations were found interposed between spots of SV2 stain representing neighboring clusters of synaptic vesicles (Fig. 3). These data suggest that the bands of actin stain observed at target-deprived synaptic sites are not localized at release sites.

F-actin associates with spectrins to form a two-dimensional lattice beneath the cell membrane (Bennett and Gilligan, 1993; Beck and Nelson, 1996). Immunohistochemical analysis of $\beta$-fodrin, a member of the spectrin family, at the target-deprived synaptic sites revealed that it has a distribution similar to that of F-actin; there were bands of $\beta$-fodrin stain (Fig. $4 A-C$ ) that did not overlap clusters of synaptic vesicles (data not shown). Further, the bands of $\beta$-fodrin stain colocalized with those of F-actin (Fig. $4 D-F$ ). This colocalization of $\mathrm{F}$-actin and $\beta$-fodrin suggests an interaction of these cytoskeletal molecules at synaptic sites. Target-deprived synaptic sites were stained only weakly by antibodies against $\alpha$-fodrin (data not shown).

\section{Cellular source of F-actin bands}

The F-actin and $\beta$-fodrin stain at nonrelease domains of synaptic sites may be derived from either the nerve terminal or the terminal Schwann cell or both (see Fig. 1). Because terminal Schwann cell processes occasionally encircle the nerve terminal in nonrelease 

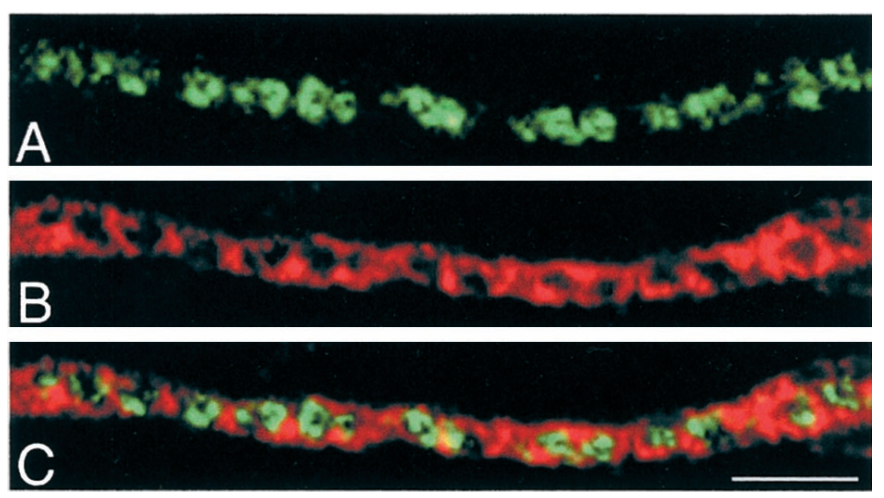

Figure 3. The majority of F-actin stain is external to synaptic vesicle clusters. A target-deprived synaptic site is stained with SV2 $(A)$ to mark synaptic vesicle clusters and with phalloidin $(B)$ to mark F-actin. $C$, The merged pseudocolor images reveal that the bands of actin are located between synaptic vesicle clusters rather than at the release sites. Scale bar, $5 \mu \mathrm{m}$.

areas, bundling of cytoskeletal molecules in these processes could produce the observed bands of $\mathrm{F}$-actin and $\beta$-fodrin stain in wholemount preparations. To assess the contribution of terminal Schwann cells to this pattern of cytoskeletal stain, we denervated target-deprived preparations for 2 weeks, a time sufficient for the nerve terminal to be phagocytized. Although denervated targetdeprived synaptic sites remained stained by phalloidin, the pattern of stain was strikingly different; no bands of actin were observed (Fig. 5A,B). Similarly, denervation resulted in the loss of the banded pattern of $\beta$-fodrin stain (Fig. $5 C$ ). Although the persistence of $\mathrm{F}$-actin and $\beta$-fodrin stain at denervated synaptic sites demonstrates that both are components of the terminal Schwann cell cytoskeleton, the change in the staining pattern suggests that some F-actin and $\beta$-fodrin are of neuronal origin. The absence of bands of $\mathrm{F}$-actin and $\beta$-fodrin stain after denervation, however, does not eliminate terminal Schwann cell processes as the source of the cytoskeletal stain because the array of glial processes may have been disrupted during phagocytosis of the nerve terminal.

To determine the extent of overlap between phalloidin-stained bands and terminal Schwann cells, we doubly-labeled synaptic sites with phalloidin and a monoclonal antibody, SC-1 or $2 \mathrm{~A} 12$, that marks terminal Schwann cells and their basal laminae (Tyner et al., 1996; Astrow et al., 1999). Although target-deprived synaptic sites were stained consistently by both antibodies, only occasionally was the terminal Schwann cell stain in a banded pattern (Fig. 6). When bands of stain were detected, there sometimes was overlap between the F-actin bands and the terminal Schwann cell stain. More frequently, however, diffuse terminal Schwann cell stain was present with strong bands of F-actin stain. These results suggest that F-actin bands were colocalized only occasionally with the stain of terminal Schwann cells.

To identify more accurately the cellular source of the F-actin bands, we compared the spacing and alignment of the phalloidinstained bands with other synaptic components. First, we compared the spacing of bands of F-actin stain and clusters of synaptic vesicles marked by SV2 stain at target-deprived synaptic sites. If the bands of F-actin stain are derived exclusively from the Schwann cell processes, we predicted that the phalloidin-stained bands would have a wider and more variable spacing when they were compared with the spacing of synaptic vesicle clusters (McMahan et al., 1972). This was not the case. Measurements determined that, on average, phalloidin-marked bands were separated by $1.2 \pm 0.3 \mu \mathrm{m}$ (mean \pm $\mathrm{SD} ; n=335$ measurements from 31 nerve terminals), whereas the centers of synaptic vesicle clusters were spaced at a distance of $1.4 \pm 0.3 \mu \mathrm{m}$ (mean $\pm \mathrm{SD} ; n=143$ measurements from 30 nerve terminals). These data suggest that neuronal F-actin is interposed regularly between neighboring clusters of synaptic vesicles and unlikely to be localized exclusively to the more irregularly spaced processes of terminal Schwann cells.
Further support for a neuronal origin of F-actin was obtained by comparing the distribution of phalloidin to other synaptic component markers in the $z$-plane. We found that the extent of phalloidin stain at a synaptic site was aligned with that of the synaptic vesicle marker. For each pair of stains the $z$-series of images of selected synaptic sites was resampled along a specified line drawn along the longitudinal axis of a synaptic site. This resampling analysis allowed for the extent of synaptic staining for phalloidin and different probes to be compared and viewed in an orientation like that schematically depicted in Figure 1. To demonstrate that this method will resolve structures that are not colocalized in the $z$-plane, we first determined that markers of synaptic vesicles and acetylcholine receptors were resolvable in normal muscles (Fig. 7). Although there is some overlap between the two stains because of the point spread function, it is clear that the two markers are resolvable in the $z$-plane (10 synaptic sites from three preparations). We then compared the pattern of F-actin stain with stains for either $\beta$-fodrin or synaptic vesicles. From analysis of the distribution of F-actin and $\beta$-fodrin (nine synaptic sites, two preparations) we determined that, in general, the stains for these molecules were overlapping and coextensive, consistent with an interaction between $\beta$-fodrin and F-actin. We next compared stains for F-actin and synaptic vesicles and predicted that the distribution of these stains at a synaptic site would differ in extent if the F-actin bands were derived solely from projections of terminal Schwann cells into the synaptic cleft. We observed, however, that probes for F-actin and synaptic vesicles (15 synaptic sites from six preparations) were frequently coextensive, beginning and ending at similar positions in the $z$-axis, although in alternating bands of stain consistent with our observations from en face images (see Fig. 3). The observed spacing of phalloidin-stained bands and their alignment with synaptic components suggests that F-actin is localized at nonrelease domains of nerve terminals.

\section{DISCUSSION}

Using a target-deprived synaptic preparation of frog neuromuscular junctions, we have shown that F-actin and $\beta$-fodrin are concentrated in a ladder-like pattern at synaptic sites. We demonstrate that $\mathrm{F}$-actin is concentrated in nonrelease domains of nerve terminals, between clusters of synaptic vesicles. Additional cytoskeletal stain may be derived from the cytoplasmic processes of terminal Schwann cells. This presynaptic distribution of actin suggests a cytoskeletal function independent of that proposed for tethering synaptic vesicles at release sites.

The target-deprived neuromuscular preparation used in these experiments offered a number of advantages for the analysis of the presynaptic cytoskeleton. First, release sites at the frog neuromuscular junction are organized in a linear array and are clearly distinguishable from intervening nonrelease domains. Second, imaging of the synaptic site was unobstructed by the muscle fiber cytoskeleton, allowing for whole-mount imaging of hundreds of mature synaptic sites in their entirety. Finally, the target-deprived preparation likely reflects the organization of the intact neuromuscular junction because target-deprived nerve terminals continue to release and recycle synaptic vesicles in response to stimulation (Dunaevsky and Connor, 1995). The observation that $\beta$-fodrin staining of normal neuromuscular junctions yielded the same ladder-like pattern of stain as observed in the absence of target muscle (our unpublished results) is further evidence that targetdeprived synaptic sites are representative of normal synapses.

Our initial experiments were designed to visualize the actin network postulated to emanate from release sites and serve as a scaffold to cluster synaptic vesicles (Greengard et al., 1993). Analysis of the three-dimensional distribution of F-actin filaments in relation to other synaptic markers allowed us to determine the localization of F-actin in relation to synaptic vesicle clusters. We were surprised when our results indicated that the majority of the actin at target-deprived nerve terminals was in nonrelease domains. Previous experiments, using rapid-freezing freeze-etch techniques, examined the presynaptic cytoskeleton and produced 

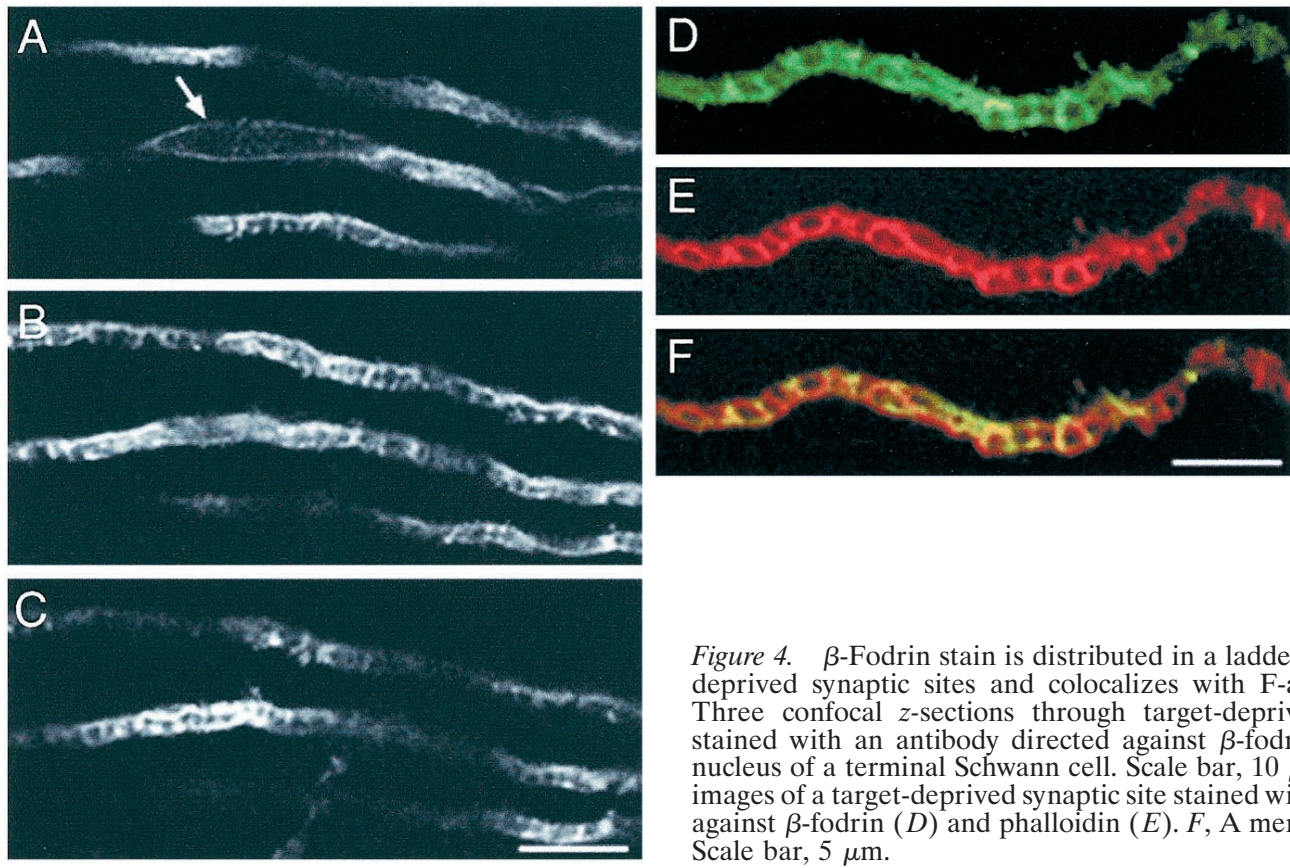

Figure 4. $\quad \beta$-Fodrin stain is distributed in a ladder-like pattern at targetdeprived synaptic sites and colocalizes with F-actin stain. $A-C$, Left, Three confocal $z$-sections through target-deprived synaptic sites are stained with an antibody directed against $\beta$-fodrin. An arrow marks a nucleus of a terminal Schwann cell. Scale bar, $10 \mu \mathrm{m}$. Right, Pseudocolor images of a target-deprived synaptic site stained with an antibody directed against $\beta$-fodrin $(D)$ and phalloidin $(E) . F$, A merged image of $D$ and $E$. Scale bar, $5 \mu \mathrm{m}$.
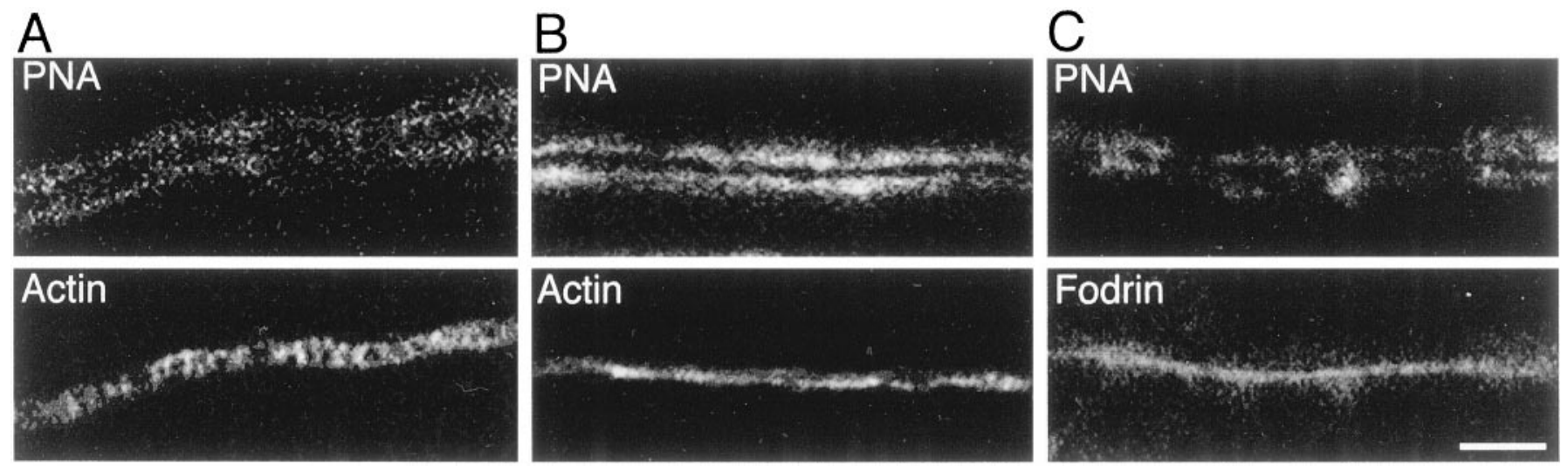

Figure 5. Bands of F-actin and $\beta$-fodrin stain do not persist after denervation of target-deprived synaptic sites. Innervated $(A)$ or 2 week denervated $(B$, $C$ ) synaptic sites are marked with peanut agglutinin $(P N A)$ and stained for actin microfilaments or $\beta$-fodrin. Scale bar, $5 \mu \mathrm{m}$.

conflicting results. Landis et al. (1988) concluded from studies of a central synapse that $\mathrm{F}$-actin is infrequent in release domains although fodrin-like filaments were observed. In contrast, Hirokawa and colleagues (1989) noted an actin meshwork at release sites of a variety of synapses as well as a lower density of filaments suggested to be fodrin. Other evidence also suggests that F-actin tethers a reserve pool of vesicles at release sites (Wang et al., 1996; Kuromi and Kidokoro, 1998). Although we may not have detected a low density of either actin or $\beta$-fodrin filaments at release domains because of the resolution limits of our techniques, our results focus attention on possible new roles of F-actin at sites removed from active zones.

Our observation that F-actin stain is focused at the synapse between release sites raised the question as to whether the actin was concentrated in the nerve terminal itself or in terminal Schwann cell processes. Our results from denervated preparations demonstrated that terminal Schwann cells contain F-actin and $\beta$-fodrin. The presence of $\beta$-fodrin in terminal Schwann cells is consistent with previous reports of fodrin localization in glial cells of the peripheral nervous system and CNS (Levine and Willard, 1981; Zagon et al., 1984, 1986). Measurements of the spacing of bands of F-actin and synaptic vesicle stain, however, suggested that F-actin is interposed regularly between neighboring clusters of synaptic vesicles and is not associated solely with terminal Schwann cell processes. Further, the extent of actin stain at synaptic sites was in line with that of synaptic vesicles, unlike similar comparisons with markers of terminal Schwann cells (data not shown). These data make it highly unlikely that the F-actin observed in nonrelease domains of synaptic sites originates exclusively from terminal Schwann cell processes.

Our data suggest a model in which a cytoskeletal matrix containing at least $\mathrm{F}$-actin and $\beta$-fodrin is positioned in nonrelease domains of the nerve terminal. What might be the role of this meshwork of actin and $\beta$-fodrin filaments? One possibility is that such a cytoskeletal network may serve as a "cage" to restrict synaptic vesicles to release sites (Betz and Henkel, 1994). Such a structure may account for the observation that FM1-43-stained clusters of synaptic vesicles did not change shape during depolarization (Betz and Bewick, 1992; Betz et al., 1992). Interestingly, treatment with cytochalasin $\mathrm{D}$, an inhibitor of actin polymerization, did not alter the distribution of synaptic vesicles at frog neuromuscular junctions (Betz and Henkel, 1994). Similarly, the pattern of FM1-43 staining in nerve terminals pretreated with cytochalasin D (Job and Lagnado, 1998) was not different from control preparations. If F-actin acts to cage synaptic vesicles, these results would require that the actin meshwork at nonrelease portions of the nerve terminal be very stable and thus not vulnerable to cytochalasin D treatment (Ayscough et al., 1997). Disruption of 

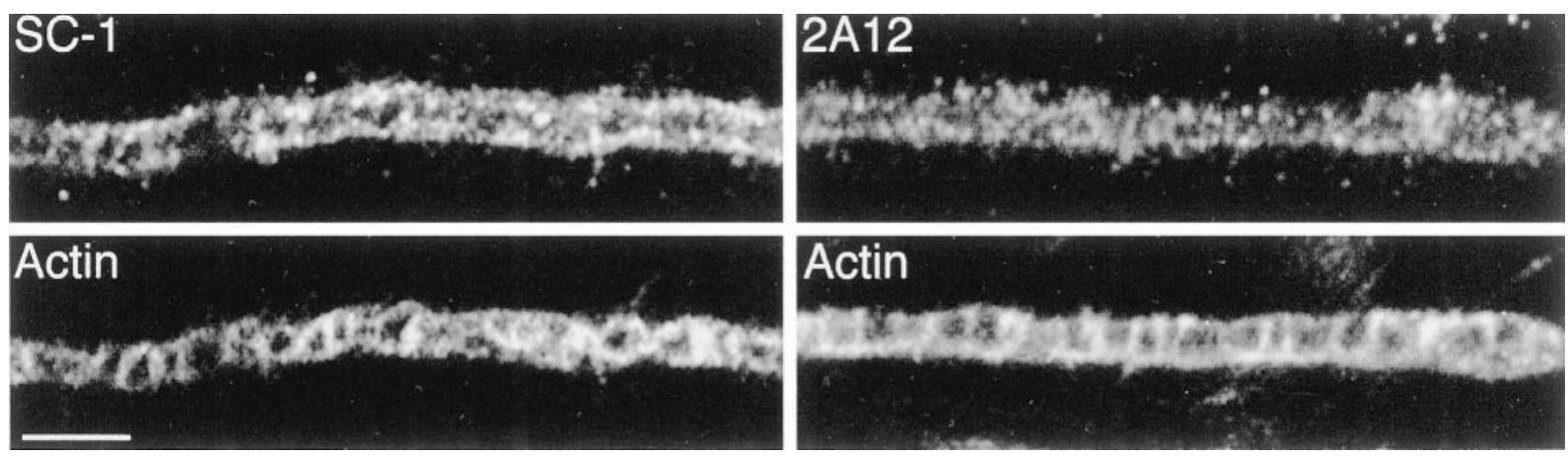

Figure 6. Terminal Schwann cell stain is not colocalized with bands of F-actin stain. Target-deprived synaptic sites were double-labeled with markers of terminal Schwann cells, either SC-1 or 2A12, and phalloidin. The terminal Schwann cell stain was rarely banded and did not overlap the bands of phalloidin stain. Scale bar, $5 \mu \mathrm{m}$.
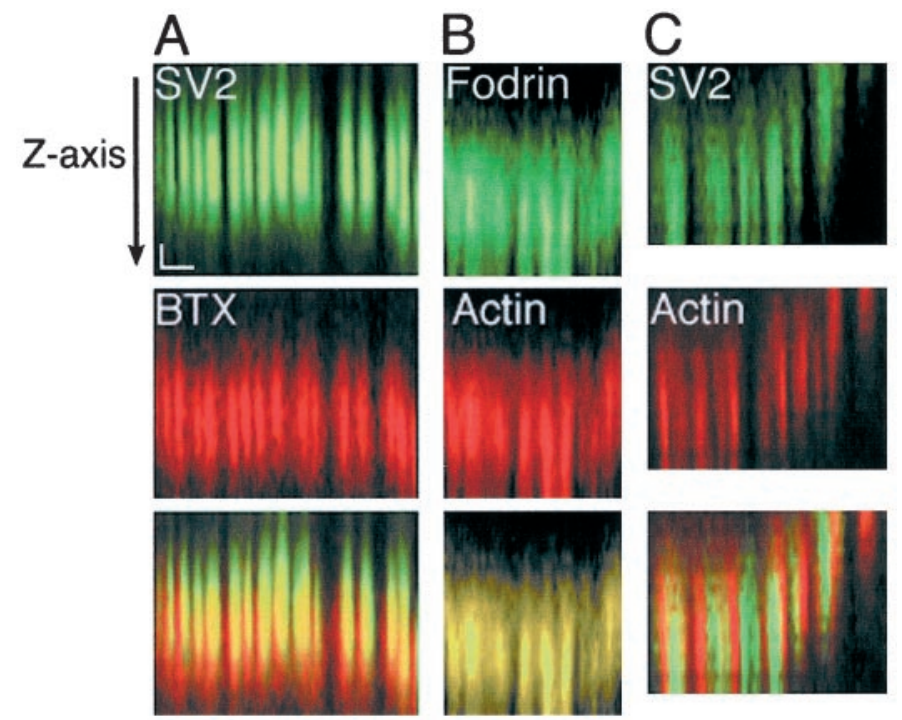

Figure 7. The distribution of F-actin at a synaptic site is coextensive with that of synaptic vesicles. Each $z$-series of confocal images was resampled along a specified line to yield a longitudinal view of a synaptic site. The view from top to bottom (arrow) shows a section through the nerve terminal toward the presynaptic membrane and synaptic cleft. Each column of three images $(A-C)$ shows reconstructed line scans of a synaptic site stained both with an antibody to either SV2 or $\beta$-fodrin (in green) and markers of either acetylcholine receptors $(B T X)$ or F-actin (in red). The bottom panels represent overlays of images from the pairs of stain. Markers of synaptic structures found in distinct locations $(A)$ were resolved in the $z$-axis by this method. F-actin is coextensive with a synaptic vesicle marker $(C)$ and overlaps completely with $\beta$-fodrin $(B)$; co-ordinates: $x$-axis, $3 \mu \mathrm{m} ; z$-axis, $0.5 \mu \mathrm{m}$.

vesicle clusters has been induced only by treatment with okadaic acid, a phosphatase inhibitor (Betz and Henkel, 1994; Dai and Peng, 1996) (but see Kuromi and Kidokoro, 1998), or a reduction in the synapsin concentration (Pieribone et al., 1995; Takei et al., 1995).

Because receptor-mediated endocytosis in yeast involves the rapid polymerization and depolymerization of F-actin (Kubler and Reizman, 1993; Ayscough et al., 1997) (for review, see Wendland et al., 1998), it has been proposed that F-actin may be involved in the endocytosis of synaptic vesicles (Mundigl et al., 1998; Gustafson et al., 1999). The F-actin bands we observed may represent such a cytoskeletal endocytotic structure. Observations that nerve terminals incorporate FM1-43 despite treatment with cytochalasin (Job and Lagnado, 1998; Kuromi and Kidokoro, 1998) and latrunculins (T. Jaquith, S. Desai, and E. A. Connor, unpublished results) suggest that F-actin polymerization is not required for vesicle recycling. A role for a stable lattticework of actin microfilaments in endocytosis of synaptic vesicles, however, cannot be ruled out.

Alternatively, an actin-based cytoskeletal network may serve to stabilize the nerve terminal at the neuromuscular junction. We have demonstrated previously that nerve terminals at frog neuromuscular junctions are maintained structurally and functionally at synaptic sites in the absence of target muscle fibers (Dunaevsky and Connor, 1995, 1998), but the mechanism by which nerve terminals are maintained at synaptic sites is not known. These and other data suggested that the cues that stabilize nerve terminals persist at synaptic sites after target removal and may reside in association with the terminal Schwann cell or the synaptic basal lamina (Trachtenburg and Thompson, 1997). It is intriguing to consider that a concentration of neuronal F-actin in nonrelease domains may provide structural integrity to the nerve terminal (Job and Lagnado, 1998), sometimes binding the nerve terminal to the synaptic basal lamina via the intermediary process of the terminal Schwann cell. Although the function of this neuronal actin network presently is unknown, the current models of synaptic vesicle localization and synapse stabilization can be tested further in light of this new morphological information.

\section{REFERENCES}

Astrow SH, Qiang H, Ko C-P (1999) Perisynaptic Schwann cells at neuromuscular junctions revealed by a novel monoclonal antibody. J Neurocytol 27:667-681.

Ayscough KR, Stryker J, Pokala N, Sanders M, Crews P, Drubin DG (1997) High rates of actin filament turnover in budding yeast and roles for actin in establishment and maintenance of cell polarity revealed using the actin inhibitor latrunculin-A. J Cell Biol 137:399-416.

Beck KA, Nelson WJ (1996) The spectrin-based membrane skeleton as a protein-sorting machine. Am J Physiol 270:C1263-C1270.

Bennett V, Gilligan DM (1993) The spectrin-based membrane skeleton and micron-scale organization of the plasma membrane. Annu Rev Cell Biol 9:27-66.

Bernstein BW, DeWitt M, Bamburg JR (1998) Actin disassembles reversibly during electrically induced recycling of synaptic vesicles in cultured neurons. Mol Brain Res 53:236-251.

Betz WJ, Bewick GS (1992) Optical analysis of synaptic vesicle recycling at the frog neuromuscular junction. Science 255:200-203.

Betz WJ, Henkel AW (1994) Okadaic acid disrupts clusters of synaptic vesicles in frog motor nerve terminals. J Cell Biol 124:843-854.

Betz WJ, Bewick GS, Ridge RM (1992) Intracellular movements of fluorescently labeled synaptic vesicles in frog motor nerve terminals during nerve stimulation. Neuron 9:805-813.

Brodin L, Low P, Gad H, Gustafsson J, Pieribone VA, Shupliakov O (1997) Sustained neurotransmitter release: new molecular cues. Eur J Neurosci 9:2503-2511.

Connor EA, Qin K, Yankelev H, DeStefano D (1994) Synaptic activity and connective tissue remodeling in denervated frog muscle. J Cell Biol 127:1435-1445.

Dai Z, Peng HB (1996) Dynamics of synaptic vesicles in cultured spinal cord neurons in relationship to synaptogenesis. Mol Cell Neurosci $7: 443-452$.

Dunaevsky A, Connor EA (1995) Long-term maintenance of presynaptic function in the absence of target muscle fibers. J Neurosci 15:6137-6144.

Dunaevsky A, Connor EA (1998) Stability of frog motor nerve terminals in the absence of target muscle fibers. Dev Biol 194:61-71.

Greengard P, Valtorta F, Czernik AJ, Benfenati F (1993) Synaptic vesicle phosphoproteins and regulation of synaptic function. Science 259:780-785.

Gustafson J, Low P, Brodin L, Shupliakov O (1999) Rho GTPases implicated as regulators of actin dynamics at presynaptic endocytic regions. Soc Neurosci Abstr 25:1743. 
Henkel AW, Simpson LL, Ridge RM, Betz WJ (1996) Synaptic vesicle movements monitored by fluorescence recovery after photobleaching in nerve terminals stained with FM1-43. J Neurosci 16:3960-3967.

Hirokawa N, Sobue K, Kanda K, Harada A, Yorifuji H (1989) The cytoskeletal architecture of the presynaptic terminal and molecular structure of synapsin I. J Cell Biol 108:111-126.

Job C, Lagnado L (1998) Calcium and protein kinase C regulate the actin cytoskeleton in the synaptic terminal of retinal bipolar cells. J Cell Biol 143:1661-1672.

Kelly RB (1993) Storage and release of neurotransmitters. Neuron [Suppl] 10:43-53.

Ko C-P (1987) A lectin, peanut agglutinin, as a probe for the extracellular matrix in living neuromuscular junctions. J Neurocytol 16:567-576.

Kraszewski K, Daniell L, Mundigl O, De Camilli P (1996) Mobility of synaptic vesicles in nerve endings monitored by recovery from photobleaching of synaptic vesicle-associated fluorescence. J Neurosci 16:5905-5913.

Kubler E, Reizman H (1993) Actin and fimbrin are required for the internalization step of endocytosis and yeast. EMBO J 12:2855-2862.

Kuromi H, Kidokoro Y (1998) Two distinct pools of synaptic vesicles in single presynaptic boutons in a temperature-sensitive Drosophila mutant shibire. Neuron 20:917-925.

Landis DMD, Hall AK, Weinstein LA, Reese TS (1988) The organization of cytoplasm at a presynaptic active zone of a central nervous system synapse. Neuron 1:201-209.

Letinsky MK, Fischbach GD, McMahan UJ (1976) Precision of reinnervation of original postsynaptic sites in frog muscle after a nerve crush. J Neurocytol 5:691-718.

Levine J, Willard M (1981) Fodrin: axonally transported polypeptides associated with the internal periphery of many cells. J Cell Biol 90:631-643.

Li L, Chin L-H, Shupliakov O, Brodin L, Sihra TS, Hvalby O, Jensen V, Zheng D, McNamara JO, Greengard P, Andersen P (1995) Impairment of synaptic vesicle clustering and of synaptic transmission, and increased seizure propensity, in synapsin I-deficient mice. Proc Natl Acad Sci USA 92:9235-9239.

McMahan UJ, Spitzer NC, Peper K (1972) Visual identification of nerve terminals in living isolated skeletal muscle. Proc R Soc Lond [Biol] 181:421-430.

Mundigl O, Ochoa G, David C, Slepnev V, Kabanov A, De Camilli P (1998) Amphiphysin I antisense oligonucleotides inhibit neurite outgrowth in cultured hippocampal neurons. J Neurosci 18:93-103.

Pieribone VA, Shupliakov O, Brodin L, Hilfiker-Rothenfluh S, Czernik AJ, Greengard P (1995) Distinct pools of synaptic vesicles in neurotransmitter release. Nature 375:493-497.

Porter GA, Reneck WG, Scher MG, Porter NC, Fowler VA, Bloch RJ
(1997) Two populations of $\beta$-spectrin in mammalian skeletal muscle. Cell Motil Cytoskeleton 37:7-19.

Rosahl TW, Spillane D, Missler M, Herz J, Selig DK, Wolff JR, Hammer RE, Malenka RC, Sudhof TC (1995) Essential functions of synapsins I and II in synaptic vesicle regulation. Nature 375:488-493.

Sanes JR, Marshall LM, McMahan UJ (1978) Reinnervation of muscle fiber basal lamina after removal of myofibers. J Cell Biol 78:176-198.

Scheller RH (1995) Membrane trafficking in the presynaptic nerve terminal. Neuron 14:893-897.

Sollner T, Bennett MK, Whiteheart SW, Scheller RH, Rothman JE (1993a) A protein assembly-disassembly pathway in vitro that may correspond to sequential steps of synaptic vesicle docking, activation, and fusion. Cell 75:409-418.

Sollner T, Whiteheart SW, Brunner M, Erdjument-Bromage H, Geromanos S, Tempst P, Rothman JE (1993b) SNAP receptors implicated in vesicle targeting and fusion. Nature 362:318-324.

Sudhof TC (1995) The synaptic vesicle cycle: a cascade of protein-protein interactions. Nature 375:645-653.

Takei Y, Harada A, Takeda S, Kobayashi K, Terada S, Noda T, Takahashi T, Hirokawa N (1995) Synapsin I deficiency results in the structural change in the presynaptic terminals in the murine nervous system. J Cell Biol 131:1789-1800.

Trachtenburg JT, Thompson WJ (1997) Nerve terminal withdrawal from rat neuromuscular junctions induced by neuregulin and Schwann cells. J Neurosci 17:6243-6255.

Tyner TR, Astrow SH, Morrow J, Ko C-P (1996) A probe for perisynaptic Schwann cells at frog neuromuscular junctions. Soc Neurosci Abstr 22:1724.

Wang XH, Zheng JQ, Poo MM (1996) Effects of cytochalasin treatment on short-term synaptic plasticity at developing neuromuscular junctions in frogs. J Physiol (Lond) 491:187-195.

Wendland B, Emr SD, Reizman H (1998) Protein traffic in the yeast endocytic and vacuolar protein sorting pathways. Curr Opin Cell Biol 10:513-522.

Yao YM (1988) Maintenance of axon terminals at synaptic sites in the absence of muscle fibers. In: Current issues in neural regeneration research (Gordon T, Stein RB, Smith PA, eds), pp 167-178. New York: Liss.

Zagon IS, McLaughlin PJ, Goodman SR (1984) Localization of spectrin in the mammalian brain. J Neurosci 4:3089-3100.

Zagon IS, Higbee R, Riederer BM, Goodman SR (1986) Spectrin subtypes in mammalian brain: an immunoelectron microscopic study. J Neurosci 6:2977-2986.

Zhou D, Ursitti JA, Bloch RJ (1998) Developmental expression of spectrins in rat skeletal muscle. Mol Biol Cell 9:47-61. 